\title{
Mast Cell Deficiency Limits the Development of Chronic Rhinosinusitis in Mice
}

\author{
Xiaoyang Hua, MD, PhD', Warren C. Naselsky, BS², Corey M. Jania, BS', \\ Kelly D. Chason, BS ${ }^{2}$, Julianne J. Huang, BS ${ }^{2}$, Claire M. Doerschuk, MD², \\ Scott M. Graham, MD', Brent A. Senior, MD $^{3}$, and Stephen L. Tilley, MD ${ }^{2,4}$
}

\begin{abstract}
Background: Chronic rhinosinusitis (CRS) is one of the most common chronic diseases in adults in both developing and developed countries. The etiology and pathogenesis of CRS remain poorly understood, and the disease is refractory to therapy in many patients. Mast cell activation has been demonstrated in the sinonasal mucosa of patients with CRS; however, the specific contribution of mast cells to the development and pathogenesis of this disease has not been established.

Objective: The objective of this study was to investigate the role of mast cells in the development of CRS.

Methods: $\mathrm{C} 57 \mathrm{BL} / 6$ wild-type and $\mathrm{C} 57 \mathrm{BL} / 6-\mathrm{Kit}^{\mathrm{W} \text {-sh/W-sh }}$ mast cell-deficient mice were immunized by intraperitoneal allergen injection and subsequent chronic low dose intranasal allergen challenges. The sinonasal phenotypes of these groups were then evaluated and compared to saline-treated controls using radiologic, histologic, and immunologic methods.

Results: Wild-type mice exposed to chronic intranasal allergen developed many features seen in human CRS, including mucosal thickening, cystic changes, polyp development, eosinophilia, goblet cell hyperplasia, and mast cell activation. In contrast, sinonasal pathology was significantly attenuated in mast cell-deficient mice subjected to the same chronic allergen protocol. Specifically, tissue eosinophilia and goblet cell hyperplasia were reduced by approximately $50 \%$ compared to wild-type levels. Surprisingly, none of the mast cell-deficient mice subjected to chronic allergen challenge developed cystic changes or polypoid changes in the nose or sinuses.

Conclusions: These data identify a critical role for mast cells in the development of many features of a mouse model of eosinophilic CRS, suggesting that therapeutic strategies targeting mast cells be examined in humans afflicted with this disease.
\end{abstract}

\section{Keywords}

mast cell, rhinosinusitis, nasal polys, mouse model, and pathogenesis

\section{Introduction}

Chronic rhinosinusitis (CRS) is a common chronic disease mainly affecting the sinonasal mucosa. Chronic rhinosinusitis causes multiple local and systemic symptoms and affects the prognosis and progression of other chronic diseases including asthma. ${ }^{1}$ Chronic rhinosinusitis is one of the major diseases adversely affecting the quality of life in the US, imposing an enormous economic burden on society. ${ }^{1}$ According to its clinical and pathological features, CRS is classified into 2 major categories: CRS without nasal polyps (CRSsNP) and CRS with nasal polyps (CRSwNP). It is believed that CRSsNP and CRSwNP represent two different disease entities. The pathogenesis of both remains poorly understood.

The local infiltration of inflammatory cells, including eosinophils, mast cells, and $\mathrm{T}$ cells, and their released mediators are thought to be central to the pathophysiology of CRSwNP..$^{1-3}$ While eosinophils are the major cell type found in most
CRSwNP specimens in Western countries, the infiltration and activation of mast cells in CRSwNP have also been observed. For instance, Carney et $\mathrm{al}^{4}$ reported that mast cell numbers were

\footnotetext{
'Department Otolaryngology-Head and Neck Surgery, University of lowa Hospitals and Clinics, lowa City, lowa, USA

${ }^{2}$ Department of Medicine, Division of Pulmonary and Critical Care Medicine, University of North Carolina at Chapel Hill, Chapel Hill, North Carolina, USA

${ }^{3}$ Department of Otolaryngology Head Neck Surgery, University of North Carolina at Chapel Hill, Chapel Hill, North Carolina, USA ${ }^{4}$ Center for Environmental Medicine, Asthma, and Lung Biology, University of North Carolina at Chapel Hill, Chapel Hill, North Carolina, USA
}

\section{Corresponding Author:}

Stephen Tilley, Department of Medicine, Division of Pulmonary and Critical Care Medicine, Center for Environmental Medicine, Asthma, and Lung Biology, University of North Carolina, 7202 Marsico Hall, 120 Mason Farm Road, Chapel Hill, NC 27599-7248, USA.

Email: stephen_tilley@med.unc.edu 
higher in CRS patients compared to the non-CRS control group. Other studies ${ }^{5-7}$ observed high tryptase levels in nasal secretions from subjects with CRSwNP compared with a non-CRS control group, indicating mast cell activation in nasal polyps. A recent study showed a histologic type change in mast cells in human nasal polyps, from mucosal type mast cells to connective tissue mast cells. ${ }^{8}$ While these data suggest that mast cells may play a role in CRS pathogenesis, mechanistic studies showing that mast cells are critical intermediaries for disease development, to the best of our knowledge, are lacking.

Mouse models have been widely used to dissect the pathogenesis of many human diseases. Not only are many different inbred laboratory mouse strains with a well-defined genetic composition available to the researcher, but these lines can be further manipulated to alter the expression of specific genes using both transgenic approaches and by the introduction of very specific changes into mouse embryonic stem cells. These genetically altered mutant mice have allowed us to carry out in-depth studies to define the roles for specific genes, proteins, and mediators in disease genesis and development both in vivo and in vitro. ${ }^{9}$ Currently, mouse models of CRS are much less developed compared to models of other human diseases. ${ }^{10}$ Widely accepted mouse models with CRSwNP remain lacking. ${ }^{10,11}$ In this report, we describe a new mouse model that recapitulates many features of human CRSwNP and used this model to specifically define the role of mast cells in this type of CRS.

\section{Material and Methods}

\section{Animals}

All animal care and experimental procedures used in this investigation were approved by the Institutional Animal Care and Use Committee of the University of North Carolina at Chapel Hill (UNC-CH). Wild-type C57BL/6 mice were purchased from the Jackson Laboratory. C57BL/6 Kit ${ }^{\text {W-sh } / W-s h ~}$ mast cell-deficient mice were provided by Dr Beverly Koller in the Department of Genetics at UNC-CH. All mice were bred in our animal facility and housed under pathogen-free conditions with 12-hour day and night switch. The mice used in the study were females 8 to 12 weeks of age at study onset and 5- to 6-month-old females at the time of harvesting. Food and water were provided ad lib for all mice throughout the duration of the study.

\section{Mouse Model of CRS}

Wild-type C57BL/6 mice and age-matched C57BL/6 $\mathrm{Kit}^{\mathrm{W}-\mathrm{sh} / \mathrm{W} \text {-sh }}$ mast cell-deficient mice were immunized by intraperitoneal injection with $50 \mu \mathrm{g}$ ovalbumin (OVA) in $0.1 \mathrm{ml}$ saline on days 1,4 , and 7 . Twelve days after the beginning of the sensitization, mice received 12 intranasal challenges with $20 \mu \mathrm{g}$ OVA in $20 \mu \mathrm{l}$ of saline 1 day per week for 12 consecutive weeks. Controls received $20 \mu$ of

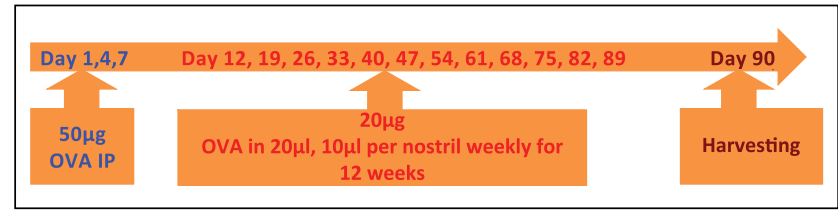

Figure I. Treatment protocol for murine CRS model.

saline without OVA. Phenotyping was performed 24 hours after the last intranasal challenge (Figure 1).

\section{MicroCT Scan}

After euthanasia by pentobarbital overdose, mice were exsanguinated and decapitated. Computed tomography (CT) scans of the nose and sinuses were obtained using a SCANCO microCT 40 scanner in the Biomedical Research Imaging Center at UNC-CH.

\section{Sinonasal Histology and Scoring}

Skulls were immediately skinned, trimmed, and fixed using $10 \%$ buffered formalin for 48 hours. The fixed skulls were then decalcified for 24 hours. After paraffin embedding, coronal sections of mouse heads were obtained at 2 levels: in front of the incisive papilla and at the level of the second palatal ridge. ${ }^{12}$ All sections were stained with $\mathrm{H} \& \mathrm{E}$, PAS-AB (goblet cells), and Toluidine blue (mast cells) stains. For quantitation of inflammation, eosinophil, goblet cell, and mast cells numbers in the lateral sinuses were counted using an inverted microscope (Nikon Eclipse Ti; Niko, Tokyo, Japan). The length of the epithelial layer in the lateral sinuses (between the tips of the maxillo- and nasoturbinates) was also measured. Cell numbers were expressed as counts per centimeter epithelium. The epithelial heights were obtained by randomly measuring 30 ciliated epithelial cells in the lateral sinuses. The examiners were blinded to the genotypes and treatments of individual mice. Basement membrane stain was performed with a standard silver technique following the manufacturer's protocol (Newcomer Supply, Middleton, Wisconsin, USA).

\section{Serum Histamine Levels}

Mouse blood was collected by cardiac puncture and serum extracted by centrifuging the blood at $14000 \mathrm{~g}$ for $15 \mathrm{~min}$ utes. Histamine levels were determined by enzyme immunoassay (SPI-BIO, Massy, France) according to manufacturer's protocol.

\section{Data Analysis}

All analyses were conducted using SPSS software. ANOVA was used to identify the difference among groups. Least significant difference was used for multiple comparisons. 
Table I. Nasal polyp presence in the lateral sinuses and/or maxillary sinuses.

\begin{tabular}{llll}
\hline Nasal Polyps & NS-WT & OVA-WT & OVA-MC $^{/-}$ \\
\hline Present & 0 & 4 & 0 \\
Absent & 6 & 2 & 6 \\
Prevalence of & 0 & 66.6 & 0 \\
$\quad$ nasal polyps (\%) & & & \\
\hline
\end{tabular}

Abbreviations: NS-WT, normal saline-treated wild-type mice; OVA-WT, ovalbumin-treated wild-type mice; OVA-MC ${ }^{-/-}$, ovalbumin-treated mast cell deficient mice.

\section{Results}

\section{Polypoid Change in the Lateral Sinuses and Maxillary Sinuses}

Since the major anatomic sites where human CRSwNP inflammation exists are the sinuses and middle meatus, in this mouse model we only analyzed the histologic changes in the lateral sinuses (which is equivalent to human middle meatus) and maxillary sinuses. ${ }^{13}$ As shown in Table 1 , four out of six OVAtreated wild-type mice (OVA-WT) developed polypoid structures in the lateral sinuses and/or maxillary sinuses. In contrast, none of the normal saline-treated wild-type mice (NS-WT) or OVA-treated mast cell deficient mice $\left(\mathrm{OVA}-\mathrm{MC}^{-1-}\right)$ exhibited polyps in the nasal cavity or sinuses. Figures $2 \mathrm{~A}$ through $2 \mathrm{C}$ are representative pictures to show the polypoid structures in the lateral sinus, medial wall of the maxillary sinus, and ostium of the maxillary sinus, respectively, of the OVA-WT mice. As shown, a central core of connective tissue similar to that in the lamina propria was usually visible in these polypoid structures, depending on the plane of the section (eg, Figure 2A). Lined by respiratory epithelium, these polypoid structures exhibited an undulated basement membrane (H\&E: Figures 2A-2C; silver stain: Figure 2D, to show the basement membrane) and parenchymal eosinophil infiltration (arrows in Figures 2A-2C). In addition, cystic changes that are frequently observed in human CRSwNP were also observed in the lateral sinuses of OVA-WT but not NS-WT or OVA-MC ${ }^{-/}$mice (Figure 2E); robust eosinophilia was observed in the lamina propria as indicated by the arrows (Figure 2A, B, C and E).

\section{Sinonasal MicroCT scan}

Similar to humans with CRS, mucosal thickening was observed in the middle turbinates and lateral sinuses of OVA-WT mice by MicroCT scan. Mucosal thickening was significantly less in OVA-MC ${ }^{-/ /}$mice (Figure 3).

\section{Eosinophilia in the Lateral Sinuses}

Eosinophilia is a cardinal feature of CRSwNP in Western countries. ${ }^{3}$ We therefore evaluated eosinophil infiltration in the lamina propria and epithelial layers of the lateral sinuses. In order to quantify the eosinophilia, eosinophil numbers were counted in the entire lateral sinuses of the NS-WT, OVA-WT, and OVA-MC ${ }^{-/-}$mice. The density of extravascular eosinophils in the lateral sinuses was then calculated as described in Materials and Methods. As shown in Figure 4A, while NS-WT mice were almost devoid of extravascular eosinophils in the lateral sinuses $(0.69 \pm 0.27$ counts/ $\mathrm{cm})$, wild-type mice treated with intranasal OVA exhibited robust extravascular eosinophilia in both the lamina propia and intraepithelial layers in the lateral sinuses $(79.6 \pm 6.3$ counts/cm; Figure 4B). Eosinophilia in the sinonasal mucosa was reduced by approximately $50 \%$ in mast celldeficient mice $(37.3 \pm 5.5$ counts $/ \mathrm{cm})$.

\section{Goblet Cell Hyperplasia and Epithelial Cell Hypertrophy}

Goblet cell hyperplasia was evaluated in the lateral sinuses of OVA-mice and saline controls (Figure 5A). In the control mice, goblet cells were confined to the nasal septum, especially on the vomeronasal organs (VNO); the lateral sinuses were largely devoid of goblet cells $(0.95 \pm 0.3$ counts $/ \mathrm{cm}$, and E Figure A). Significant goblet cell hyperplasia was observed in the lateral sinuses of the OVA-WT mice ( $33.5 \pm$ 2.3 counts $/ \mathrm{cm}$, and E Figure B), while goblet cell hyperplasia was significantly attenuated in OVA-MC ${ }^{-/ 2}$ mice $(12.2 \pm$ 2.3 counts $/ \mathrm{cm}$; and E Figure C).

Epithelial cell hypertrophy as the result of OVA treatment was evaluated by measuring the average height of ciliated epithelial cells in the lateral sinuses of the NS-WT, OVA-WT, and OVA-MC ${ }^{-/-}$mice. As shown in Figure 5B, the OVA-WT mice exhibited epithelial cell hypertrophy following intranasal OVA challenge which was not observed in saline controls. In contrast, epithelial height in OVA-MC ${ }^{-/ 2}$ mice was significantly lower than that observed in OVA-WT mice and no different than epithelial height in NS-WT control animals.

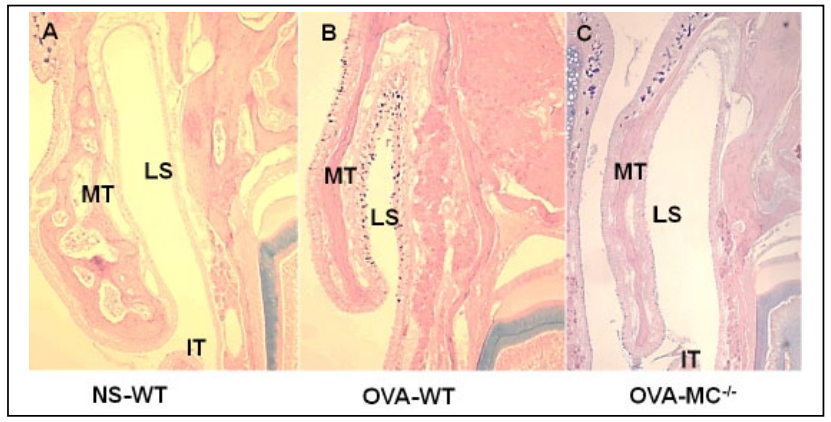

Figure E. Goblet cell hyperplasia in the lateral sinuses of representative mice in the NS treated wild-type (control, NSWT), OVA-treated wild-type (OVA-WT) and OVA- treated mast cell deficient (OVA-MC-/-) groups (AB-PAS stain). $M T$, middle turbinate; IT: inferior turbinate; LS, lateral sinus (equivalent to human middle meatus). 

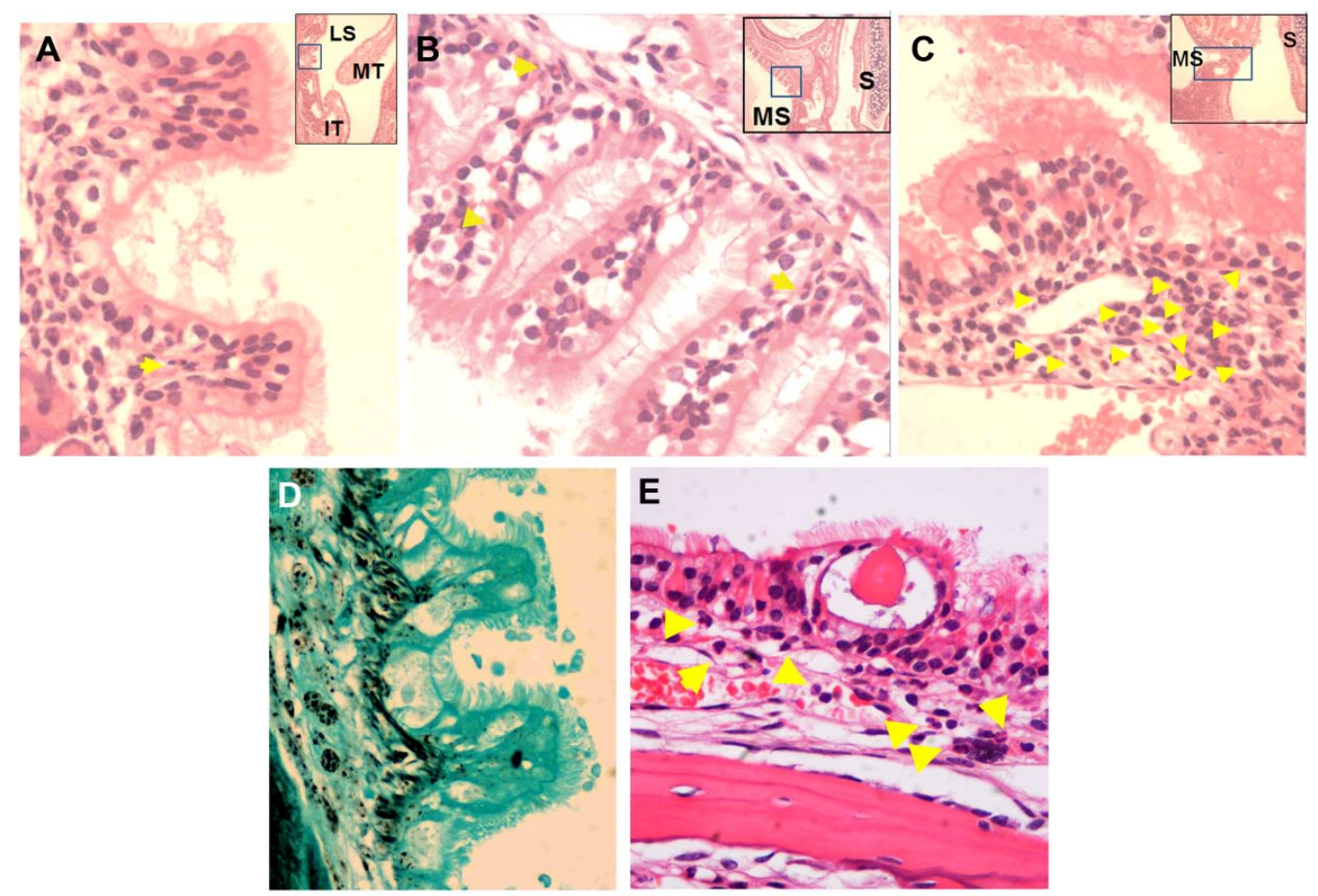

Figure 2. Nasal polyps in the lateral and maxillary sinuses of OVA-treated wild-type mice. (A) Polyps in the lateral sinus. (B) Polyps in the maxillary sinus. (C) Polyps in the ostium of maxillary sinus (40x, H\&E). (D) Basement membrane undulation (40x, silver stain). (E) Formation of cystic changes (40x). Arrows indicate eosinophils. IT, inferior turbinate; also termed maxilloturbinate in rodents; LS, lateral sinus (equivalent to human middle meatus); MS, maxillary sinus; MT, middle turbinate; also termed nasoturbinate in rodents; $\mathrm{S}$, nasal septum; OVA, ovalbumin.

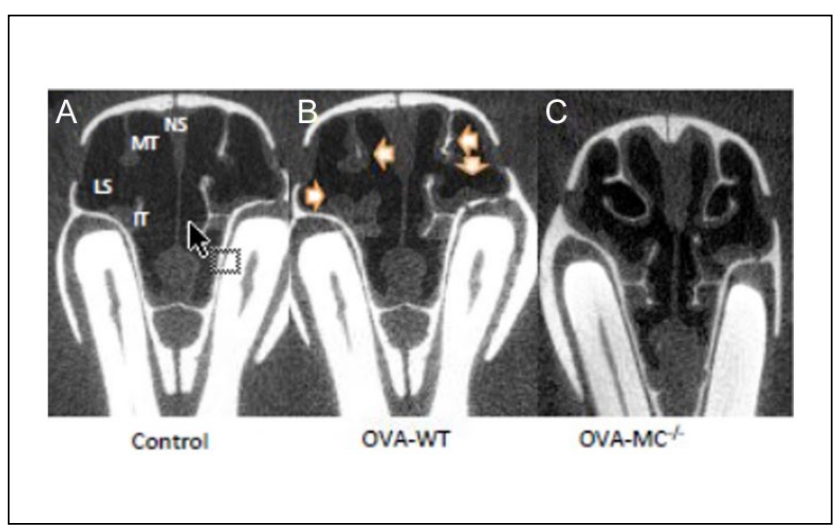

Figure 3. MicroCT scans of murine nose and sinuses. Representative computed tomography (CT) scans of the nose and sinuses in the (A) normal saline-treated wild-type (NS-WT), (B) OVA-treated wild-type (OVA-WT), and (C) OVA-treated mast cell deficient mice (OVA-MC $\mathrm{C}^{-/}$) were obtained using a SCANCO microCT 40 scanner. Mucosal thickening (arrows) in both the nasoturbinates and lateral sinuses were evident in the OVA-treated wild-type but not in the control or the OVA$\mathrm{MC}^{-/-}$mice.

\section{Mast Cell Histology and Serum and Histamine Levels}

Mast cell numbers in the sinonasal mucosa was measured histologically. No difference was observed between the NS-WT and OVA-WT groups (Figure 6A). However, serum histamine levels were higher in OVA-WT mice compared with both control mice and OVA-MC ${ }^{-/-}$mice (Figure 6B). In addition, in OVA-WT mice, mast cells were frequently observed to reside closer to the epithelial layers (data not shown).

\section{Discussion}

Mouse models have been widely used in biomedical research to dissect disease pathogenesis. In this study, we used a chronic, low dose, intranasal allergen challenge protocol and have successfully reproduced many clinical and pathological features of human CRSwNP in mice. These features include the presence of polypoid changes in the lateral sinuses and maxillary sinuses, goblet cell hyperplasia, 


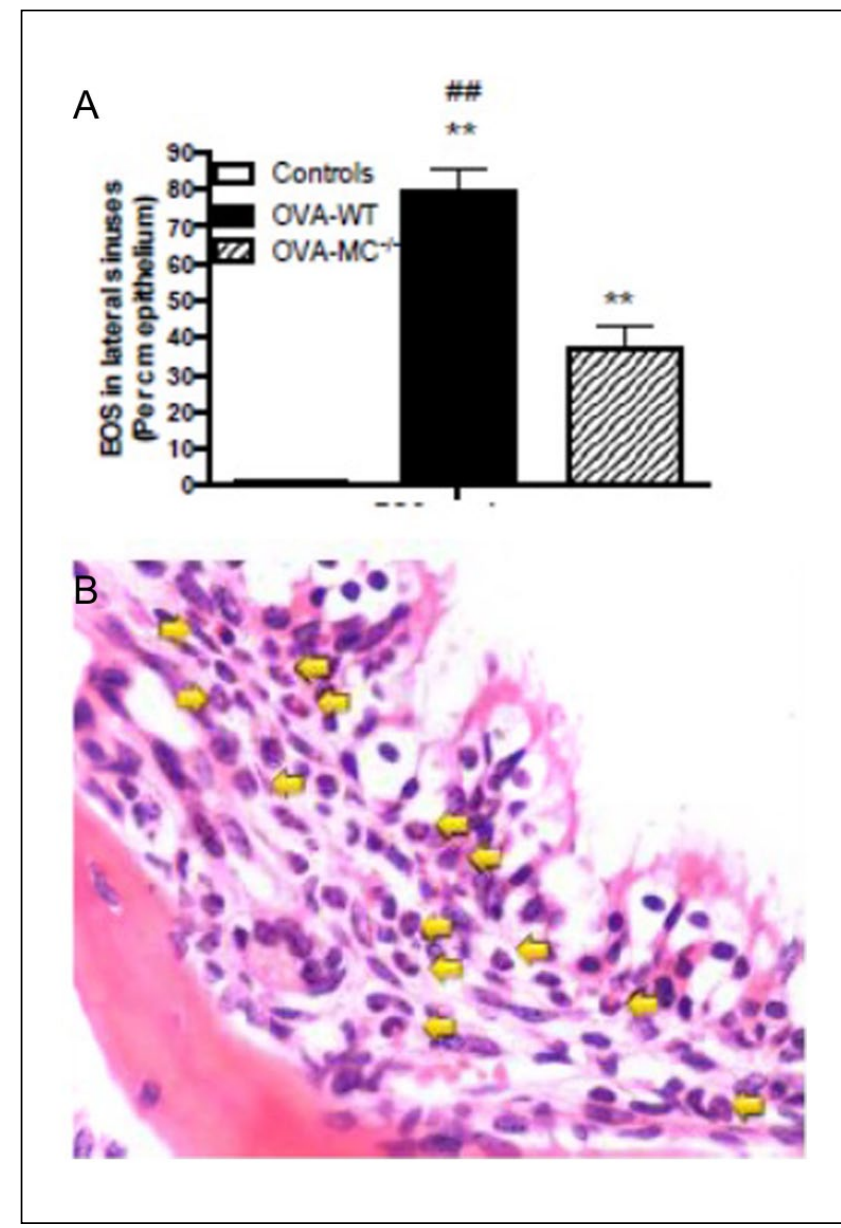

Figure 4. Eosinophil infiltration. (A) Eosinophil density in the lateral sinuses of normal saline-treated wild-type (NS-WT), ovalbumin-treated wild-type (OVA-WT), and OVA-treated mast cell-deficient (OVA-MC ${ }^{-1}$ ) mice was expressed as counts per $\mathrm{cm}$ epithelial cells. ${ }^{* * P}<.01$ versus NS-WT; \#\#P $<.01$ versus OVA$M^{-/-} . N=6$ per group. The examiners were blinded to the genotypes and treatments of individual mice. (B) Lateral sinus of representative OVA-WT mouse to show the robust infiltration of eosinophils in both the lamina propria and intraepithelial layers. Arrows indicate eosinophils.

epithelial cell hypertrophy, eosinophilia, and mast cell activation. The polypoid structures were only observed in the lateral sinuses (equivalent to the middle meatus in humans) and maxillary sinuses, which is consistent with the observations in humans that the middle meatus and middle turbinates are the major anatomic sites affected by CRSwNP. In addition, cystic changes were frequently present in the sinuses of this animal model.

Our data show that mast cell deficiency substantially limits the development of several key phenotypes of CRS, including the formation of nasal polyps, suggesting a critical role for mast cells in CRSwNP development. In humans, enhanced mast cell activity has been reported in CRSwNP. Mast cell autocoids including tryptase have been shown to be

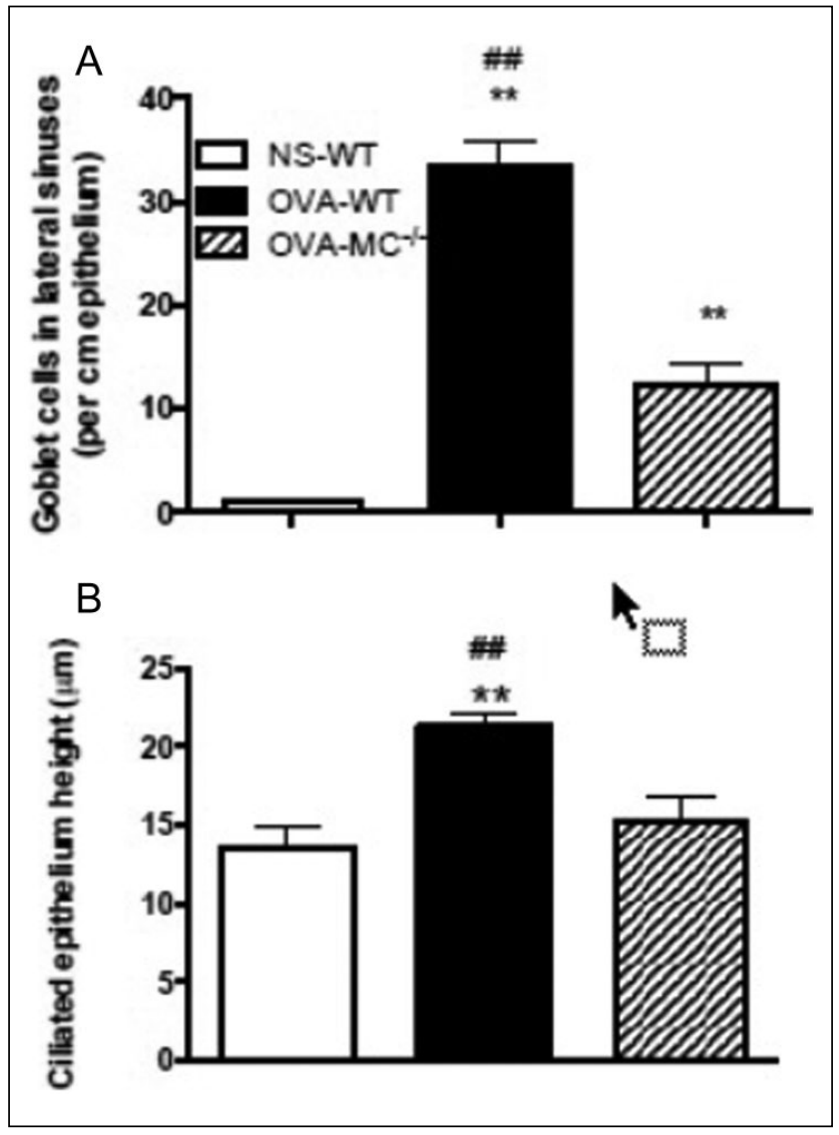

Figure 5. Goblet cell hyperplasia and epithelial hypertrophy. (A) Goblet cell numbers in the lateral sinuses were counted and normalized to the length of the lining epithelium. (B) Epithelial cell height was obtained by randomly measuring 30 ciliated epithelial cells in the lateral sinuses. The examiners were blinded to the genotypes and treatments of individual mice. **P $<.01$ versus NS-WT. \#\#P $<.01$ versus ovalbumin-treated mast cell-deficient (OVA-MC $\mathrm{MC}^{-1}$ ) mice. $\mathrm{N}=6$ per group.

high in nasal polyp homogenates and nasal secretions from patients with CRSwNP. ${ }^{4,7,14}$ Moreover, a recent clinical trial has also shown that anti-IgE treatment provided a better therapeutic effect than anti-IL-5, suggesting that mast cells may play a more important role than eosinophils in CRSwNP pathogenesis. ${ }^{11,15-17}$ While these human studies suggest that mast cells are activated in patients with CRSwNP, this study, to the best of our knowledge, is the first to suggest that mast cells are critical to disease development.

Eosinophil infiltraton of tissues is medated by eosinophilspecific cytokines/chemokines, including IL-5 and eotaxin. Several cell types secrete these eosinophil chemoattractants including T-cells and activated mast cells. Consistent with this paradigm, we observed a 50\% reduction in tissue eosinophilia in OVA-treated mast cell-deficient mice, suggesting that mast cells may be an important source of eosinophil chemoattractants driving the tissue eosinophilia seen in some CRS patients. 


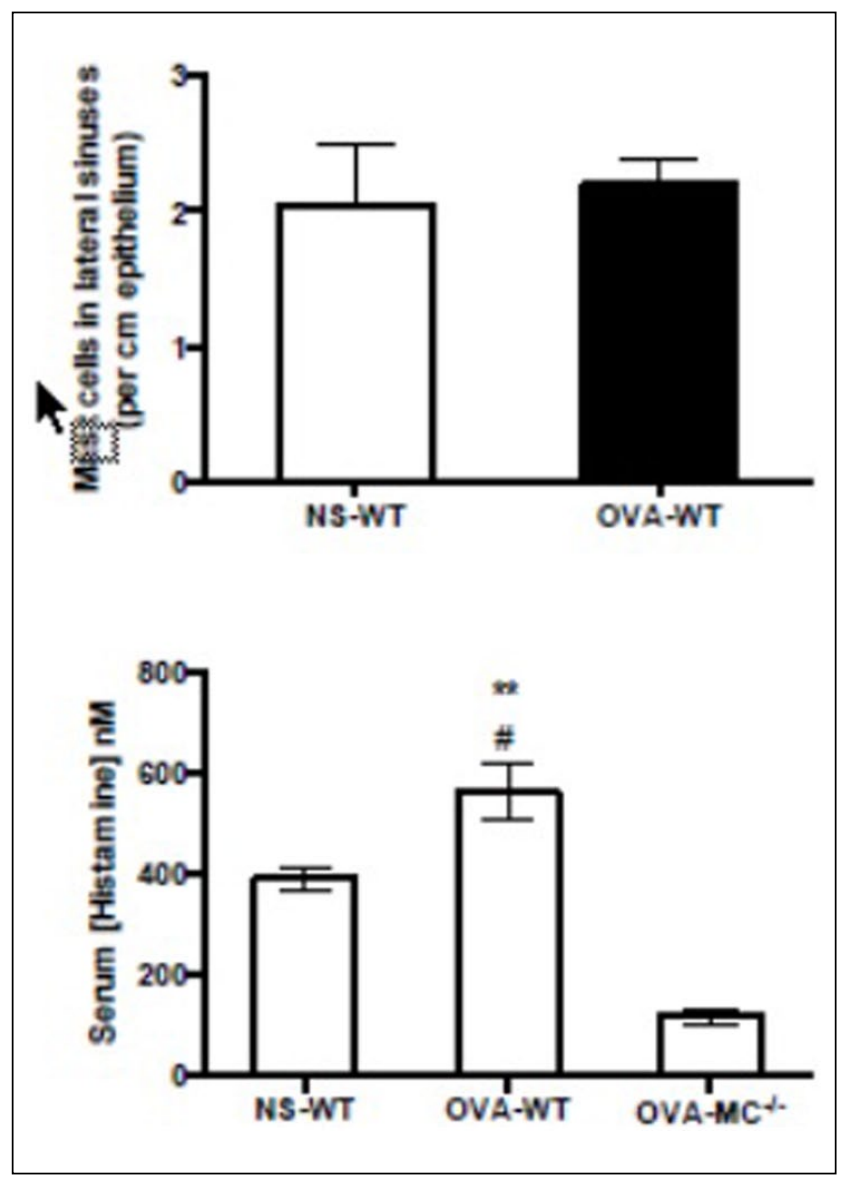

Figure 6. (A) Mast cell numbers in the lateral sinuses of the normal saline-treated wild-type (control, NS-WT) and ovalbumin-treated wild-type (OVA-WT) mice. $P>.05$. (B) Serum histamine levels. \#P $<.05$ versus NS-WT mice. $* * P<.0$ I versus OVA-MC $\mathrm{MC}^{-1-}$ mice. $\mathrm{N}=6$ per group.

In the OVA-treated wild type mice, there were one-third of mice that did not develop polypoid structures in the nose and sinuses. One possible explanation is that only two coronal sections of each mouse head were examined. In addition, since CRSwNP is a multifactorial disease, there could be inter-mouse difference in their response to intranasal OVA challenge. However, the difference between OVA-WT and OVA-MC $\mathrm{MC}^{-/-}$mice strongly suggests that mast cells play a critical role in the development of nasal polyps.

In this study, we used a chronic, low dose, intranasal allergen challenge protocol without adjuvant to generate the mouse model of CRS. It is well known that using immune adjuvant, for instance, aluminum hydroxide, generates very strong Th2 polarized airway inflammation in mice. This artificial Th2 polarization bypasses the role of mast cells in disease pathogenesis. ${ }^{13,18,19}$ In published CRS research, many mouse models were generated with the use of immune adjuvants. ${ }^{20-24}$ While these studies have provided important information on CRS pathogenesis, it remains unknown if they can accurately reflect human CRS pathophysiology given the bypass of mast cell contribution. Mast cells can be activated both acutely and chronically. ${ }^{25}$ While acute activation of mast cells is central to the pathophysiology of anaphylactic reactions, including asthma attacks, chronic activation of mast cells, including so-called piecemeal activation, plays an important role in the progression of chronic inflammation, including tissue remodeling. ${ }^{25,26}$ For instance, $\mathrm{Yu}$ et $\mathrm{al}^{27,28}$ used a chronic low dose intranasal allergen treatment without aluminum to generate mouse model of asthma in order to better simulate the natural allergen exposure in humans. They observed that mast cells were the major contributor to many cardinal pathologic features of chronic asthma, including airway hyperresponsiveness, airway remodeling, goblet cell hyperplasia, over-expression of mucin genes, eosinophilia and $\mathrm{T}$ cell recruitment. ${ }^{28}$

One limitation to our study is that we have developed a purely allergen-driven CRS model while clinically it remains controversial whether allergen alone can drive the development of CRS since other factors such as sinonasal bacteria are believed to play a role. The fact that our allergen-dependent model demonstrates many pathological features of CRS suggests that without introducing artificial or bacterial adjuvants, allergen alone can drive disease pathogenesis, at least in mice.

While we observed mast cell activation and migration toward the epithelium (data not shown), we failed to observe increased infiltration of mast cells in the nose and sinuses of this CRS model. In human studies, whether patients with CRSwNP have increased sinonasal mast cell infiltration is still controversial. While some human studies have shown slightly increased mast cell numbers in nasal polyp tissues, several groups reported enhanced mast cell activation but not increased mast cell infiltration, similar to the phenotype of our mouse model. A recent study further showed that mast cell distribution patterns and histological types were altered in human CRSwNP, suggesting that mast cells contribute to the disease pathogenesis through the alteration of their activities rather than simply through increased local infiltration. ${ }^{8}$ In addition, mast cells can be activated by many endogenous and exogenous factors and mediators via both IgE-dependent and IgE-independent pathways. ${ }^{25} \mathrm{~A}$ clinical trial with the anti-IgE antibody omalizumab showed only moderate efficacy in reducing nasal polyp size and improving the Lund Mackay CT scores in patients with CRSwNP, ${ }^{16}$ suggesting that mast cells in the nose and sinuses of patients with CRSwNP may also be activated by non-IgE mediated mechanisms.

\section{Conclusions}

In summary, we have successfully generated a mouse model that reproduces many features of human CRSwNP. To the best of our knowledge, this is the first study to show that mast cells are critical for the full development of many 
histopathological features of CRSwNP. We believe that this model will become a useful tool to advance our understanding of the etiology and pathophysiology of CRSwNP and to test new therapies. In addition, our findings suggest that mast cell-mediated biology may be central to CRSwNP pathophysiology and that specifically inhibiting both IgEdependent and IgE-independent pathways of mast cell activation may be of therapeutic value for this common upper airway disease.

\section{Acknowledgments}

The authors would like to thank Kimberly Burns from the Cystic Fibrosis Center at University of North Carolina for the preparation of the sinonasal histology and Dr Stanley Perlman at the University of Iowa for reading and editing the manuscript.

\section{Declaration of Conflicting Interests}

The author(s) declared no potential conflicts of interest with respect to the research, authorship, and publication of this article.

\section{Funding}

The authors disclosed receipt of the following financial support for the research, authorship, and/or publication of this article: This work was made possible by funding from NIH grants HL071802 (S.L.T), U19AI077437 (D.B. Peden), 1R21AI096139-01A1 (X.H and S.L.T), 2T32DC000040-21 (B.J Gantz); AAO-HNS/Cook Medical CORE Grant 314113 (X.H) and AAO-HNS/AAOA Core Grant 92997 (X.H); and the NC TraCs grant 50KR20926 (X.H). There is no other financial support to disclose.

\section{References}

1. Meltzer EO, Hamilos DL, Hadley JA, et al. Rhinosinusitis: establishing definitions for clinical research and patient care. J Allergy Clin Immunol. 2004;114:155-212.

2. Wood AJ, Douglas RG. Pathogenesis and treatment of chronic rhinosinusitis. Postgrad Med J. 2010;86:359-364.

3. Fokkens W, Lund V, Mullol J. European position paper on rhinosinusitis and nasal polyps 2007. Rhinol Suppl. 2007;(20):1-136.

4. Carney AS, Tan LW, Adams D, Varelias A, Ooi EH, Wormald PJ. Th2 immunological inflammation in allergic fungal sinusitis, nonallergic eosinophilic fungal sinusitis, and chronic rhinosinusitis. Am J Rhinol. 2006;20:145-149.

5. Kramer MF, Burow G, Pfrogner E, Rasp G. In vitro diagnosis of chronic nasal inflammation. Clin Exp Allergy. 2004;34:1086-1092.

6. Loesel LS. Immunopathologic study of chronic sinusitis: a proposal for atopic and non-atopic IgE-activated mast cell allergic inflammation. Ann Otol Rhinol Laryngol. 2001;110:447-452.

7. Drake-Lee A, Price J. Mast cell ultrastructure in the inferior turbinate and stroma of nasal polyps. J Laryngol Otol. 1997;111:340-345.

8. Takabayashi T, Kato A, Peters AT, et al. Glandular mast cells with distinct phenotype are highly elevated in chronic rhinosinusitis with nasal polyps. J Allergy Clin Immunol. 2012;130:410-420 e415.

9. Shapiro SD. Animal models of asthma: pro: allergic avoidance of animal (model[s]) is not an option. Am J Respir Crit Care Med. 2006;174:1171-1173.
10. Wagner JG, Harkema JR. Rodent models of allergic rhinitis: relevance to human pathophysiology. Curr Allergy Asthma Rep. 2007;7:134-140.

11. Kern RC. Biologics and the treatment of chronic rhinosinusitis. J Allergy Clin Immunol. 2013;131:117-118.

12. Ostrowski LE, Yin W, Rogers TD, et al. Conditional deletion of dnaic1 in a murine model of primary ciliary dyskinesia causes chronic rhinosinusitis. Am J Respir Cell Mol Biol. 2010;43:55-63.

13. Harkema JR, Carey SA, Wagner JG. The nose revisited: a brief review of the comparative structure, function, and toxicologic pathology of the nasal epithelium. Toxicol Pathol. 2006;34:252-269.

14. Groger M, Bernt A, Wolf M, et al. Eosinophils and mast cells: a comparison of nasal mucosa histology and cytology to markers in nasal discharge in patients with chronic sino-nasal diseases. Eur Arch Otorhinolaryngol. 2013;270:2667-2676.

15. Vennera Mdel C, Picado C, Mullol J, Alobid I, BernalSprekelsen M. Efficacy of omalizumab in the treatment of nasal polyps. Thorax. 2011;66:824-825.

16. Gevaert P, Calus L, Van Zele T, et al. Omalizumab is effective in allergic and nonallergic patients with nasal polyps and asthma. J Allergy Clin Immunol. 2013;131:110-116.

17. Gevaert P, Lang-Loidolt D, Lackner A, et al. Nasal IL-5 levels determine the response to anti-IL-5 treatment in patients with nasal polyps. J Allergy Clin Immunol. 2006;118:1133-1141.

18. Bates JH, Rincon M, Irvin CG. Animal models of asthma. Am J Physiol Lung Cell Mol Physiol. 2009;297:L401-L410.

19. Takeda K, Hamelmann E, Joetham A, et al. Development of eosinophilic airway inflammation and airway hyperresponsiveness in mast cell-deficient mice. $J$ Exp Med. 1997; 186:449-454.

20. Saito H, Howie K, Wattie J, et al. Allergen-induced murine upper airway inflammation: local and systemic changes in murine experimental allergic rhinitis. Immunology. 2001;104:226-234.

21. Kim SW, Kim JH, Jung MH, et al. Periostin may play a protective role in the development of eosinophilic chronic rhinosinusitis with nasal polyps in a mouse model. Laryngoscope. 2013;123:1075-1081.

22. Lindsay R, Slaughter T, Britton-Webb J, et al. Development of a murine model of chronic rhinosinusitis. Otolaryngol Head Neck Surg. 2006;134:724-730.

23. Liang KL, Jiang RS, Wang RC, et al. Upper airway inflammation exacerbates bronchial hyperreactivity in mouse models of rhinosinusitis and allergic asthma. Int Forum Allergy Rhinol. 2013;3:532-542.

24. Sautter NB, Delaney KL, Hausman FA, Trune DR. Tissue remodeling gene expression in a murine model of chronic rhinosinusitis. Laryngoscope. 2012;122:711-717.

25. Galli SJ, Kalesnikoff J, Grimbaldeston MA, Piliponsky AM, Williams CM, Tsai M. Mast cells as "tunable" effector and immunoregulatory cells: recent advances. Annu Rev Immunol. 2005;23:749-786.

26. Bradding P. Mast cell ion channels. Chem Immunol Allergy. 2005;87:163-178.

27. Yu M, Eckart MR, Morgan AA, et al. Identification of an IFNgamma/mast cell axis in a mouse model of chronic asthma. J Clin Invest. 2011;121:3133-3143.

28. Yu M, Tsai M, Tam SY, Jones C, Zehnder J, Galli SJ. Mast cells can promote the development of multiple features of chronic asthma in mice. J Clin Invest. 2006;116:1633-1641. 